\title{
Implementation of a regional quality improvement collaborative to improve care of people living with opioid use disorder in a Canadian setting
}

Laura Beamish', Zach Sagorin ${ }^{1}$, Cole Stanley ${ }^{1,2,3}$, Krista English ${ }^{1}$, Rana Garelnabi ${ }^{1}$, Danielle Cousineau ${ }^{1,2}$, Rolando Barrios ${ }^{1,2,5^{*}}$ (D) and Jan Klimas ${ }^{1,4,6,7}$

\begin{abstract}
Background: Although opioid agonist therapy is effective in treating opioid use disorders (OUD), retention in opioid agonist therapy is suboptimal, in part, due to quality of care issues. Therefore, we sought to describe the planning and implementation of a quality improvement initiative aimed at closing gaps in care for people living with OUD through changes to workflow and care processes in Vancouver, Canada.

Methods: The Best-practice in Oral Opioid agoniSt Therapy (BOOST) Collaborative followed the Institute for Healthcare Improvement's Breakthrough Series Collaborative methodology over 18-months. Teams participated in a series of activities and events to support implementing, measuring, and sharing best practices in OAT and OUD care. Teams were assigned monthly implementation scores to monitor their progress on meeting Collaborative aims and implementing changes.

Results: Seventeen health care teams from a range of health care practices caring for a total of 4301 patients with a documented diagnosis of OUD, or suspected OUD based on electronic medical record chart data participated in the Collaborative. Teams followed the Breakthrough Series Collaborative methodology closely and reported monthly on a series of standardized process and outcome indicators. The majority of (59\%) teams showed some improvement throughout the Collaborative as indicated by implementation scores.
\end{abstract}

Conclusions: Descriptive data from the evaluation of this initiative illustrates its success. It provides further evidence to support the implementation of quality improvement interventions to close gaps in OUD care processes and treatment outcomes for people living with OUD. This system-level approach has been spread across British Columbia and could be used by other jurisdictions facing similar overdose crises.

Keywords: Opioid use disorder, Opioid agonist therapy, Methadone, Suboxone, Buprenorphine/naloxone, Retention, Quality improvement, Primary care, Substance use disorder, Mental health

\footnotetext{
* Correspondence: rbarrios@cfenet.ubc.ca

${ }^{1}$ British Columbia Centre for Excellence in HIV/AIDS (BC-CfE), St. Paul's

Hospital, 608-1081 Burrard Street, Vancouver, BC V6Z1Y6, Canada

${ }^{2}$ Vancouver Coastal Health, 520 W 6th Ave, Vancouver, BC V5Z4H5, Canada

Full list of author information is available at the end of the article
}

(c) The Author(s). 2019 Open Access This article is distributed under the terms of the Creative Commons Attribution 4.0 International License (http://creativecommons.org/licenses/by/4.0/), which permits unrestricted use, distribution, and reproduction in any medium, provided you give appropriate credit to the original author(s) and the source, provide a link to the Creative Commons license, and indicate if changes were made. The Creative Commons Public Domain Dedication waiver (http://creativecommons.org/publicdomain/zero/1.0/) applies to the data made available in this article, unless otherwise stated. 


\section{Background}

Canada, much like the rest of North America, is in the midst of an overdose crisis. Since 2016, approximately 10, 000 people have died as a result of opioid-related overdose, with the province of British Columbia (BC) reporting the highest rates of accidental opioid-related deaths [1]. The crisis in $\mathrm{BC}$ has highlighted significant gaps in care for people living with opioid use disorders (OUD).

Current Canadian clinical guidelines for the management of OUD recommend the use of opioid agonist therapies (OAT) as first line treatment [2]. A recent meta-analysis demonstrated that retention in OAT is associated with two to three times lower all-cause and overdose-related mortality in people with OUD [3]. A significant proportion of individuals with OUD reduce illicit opioid use, remain in treatment using appropriate doses of OAT, such as methadone and buprenorphine/ naloxone [4].

Delivering appropriate care and treatment for OUD is a complex process that requires collaboration among the most responsible provider (MRP) and other care providers - from screening and diagnosis to treatment and follow-up [5, 6]. The term MRP (most responsible physician/practitioner/provider), generally refers to regulated healthcare professional, who has overall responsibility for directing and coordinating the care and management of a patient at a specific point in time. While typically referring to a physician, this may include a nurse practitioner or other healthcare professional [7]. Establishing effective and proactive systems of care within which OUD treatment can be delivered is essential for treatment to be successful and sustainable. The chronic care model (CCM) is cited as an effective organizing framework for improvement initiatives [8].

Increasingly, the healthcare community is employing quality improvement (QI) frameworks to promote system-level change and address gaps in practice [9]. The Breakthrough Series (BTS) Collaborative methodology, developed by the Institute for Healthcare Improvement (IHI), is a QI approach designed to help healthcare organizations systematically close the gaps between evidence and practice [10]. Although literature reported variable success of QI approaches [11], BTS has been successfully applied in other drug treatment settings in the United States [12-14], and for other chronic diseases in Canada, including diabetes, congestive heart failure and most recently HIV/AIDS [15-17]. In 2017, the BC Centre for Excellence in HIV/AIDS (BC-CfE) - a provincial resource for HIV/AIDS care, treatment, education, research and evidence-based policy development - and Vancouver Coastal Health (VCH) launched a BTS Collaborative, titled the best-practice in oral opioid agonist therapy, or the BOOST Collaborative. The initiative aimed to systematically implement, measure and share best-practices in oral opioid OAT and improve outcomes for people living with OUD in Vancouver, Canada. The current paper sought to describe the planning and implementation of a BTS Collaborative aimed at closing gaps in care for people living with OUD through changes to workflow and care processes.

\section{Context}

British Columbia is Canada's westernmost province and has a population of approximately 4.8 million [18]. In 2016, the province declared a state of public health emergency in response to the alarming increase in the rate of opioid-related overdose deaths [19]. BC is at the epicentre of the overdose crisis in Canada with over 4000 opioid-related overdose deaths since the declaration, with over 1100 of those deaths occurring in the Vancouver region [1]. The majority of opioid-related overdose deaths occur in men aged 30-39 and disproportionately affect Indigenous and/or First Nations people of $\mathrm{BC}^{1}$. Current data from the Office of the Provincial Health Officer shows poor OAT retention rates in Vancouver, with only $42 \%$ of people who start OAT retained at six months, with this dropping to $32 \%$ at 12 months [20]. The province has launched a number of services to address the crisis, including the scale-up of naloxone distribution, the expansion and establishment of supervised consumptions sites, expanded low barrier substance use disorder (SUD) care, and improved access to treatment such as OAT [21]. Although considerable work has been done to address the crisis, the death rate remains well above historical average [1]. BC Coroners Service data shows the majority of opioid-related overdose deaths are occurring in people not retained on OAT, highlighting a key gap in care $[22,23]$. With increasing evidence that OAT can reduce overdose and all-cause mortality, Vancouver Coastal Health regional health authority the BC-CfE committed funding for a QI Collaborative to launch in September 2017. This pilot was called the Vancouver Best-practices in Oral Opioid agoniSt Therapy, or the BOOST, Collaborative.

\section{Health system organization}

The health care system in BC is predominantly funded publicly through taxation and other revenue sources [23]. In the provincial government, health care is organized into the Ministry of Health and the newly formed Ministry of Mental Health and Addiction. These two Ministries are responsible for setting the strategic direction and priorities in the province [23]. Health care funding is directed from the Ministry of Health to five regional health authorities who deliver care in their respective regions. There are also two provincial health authorities responsible for specific programs and populations across the province [23]. In BC, pharmaceutical 
drugs are not publicly funded, however the Ministry of Health funds OAT (methadone, buprenorphine/naloxone, and slow release oral morphine) for populations who show both a medical and financial need [24].

The majority of care for people with OUD in Vancouver is delivered in interprofessional community health centres where physicians are remunerated at a government negotiated sessional rate [25], making them the focus of this intervention. In some cases, OUD care is provided in fee-for-service private practice settings or in hospital settings for a short duration of time (induction and stabilization) [26].

\section{Methods}

\section{Intervention components}

The BOOST Collaborative followed the IHI's BTS Collaborative methodology for 18-months from September 2017 to December 2018 (see Fig. 1). The overarching aim of the BOOST Collaborative was to provide equitable access to integrated, evidence-based care to help our population of clients with OUD achieve: 95\% initiated on OAT; 95\% retained in care for $\geq 3$ months; and $50 \%$ average improvement in quality of life scores. The BC Centre for Excellence in HIV/AIDS-a provincial resource for HIV/AIDS care, treatment, education, research and evidence-based policy development-provided intervention leadership and coordination with QI support from the Practice Support Program, a program of the General Practice Services Committee at the Doctors of $\mathrm{BC}$ dedicated to in-practice QI coaching and support. The program was funded by Vancouver Coastal Health with in-kind contributions from the BC Centre for Excellence in HIV/AIDS. Delivery costs included learning sessions, educational webinars, and Collaborative staff time and travel. Vancouver Coastal Health covered the cost of staff participation and travel to learning sessions, including the required backfill.

\section{Planning and team recruitment}

In July 2017 a multi-stakeholder working group meeting was held to help shape the technical content of the

\section{ORAL OPIOID AGONIST THERAPY COLLABORATIVE METHODOLOGY}

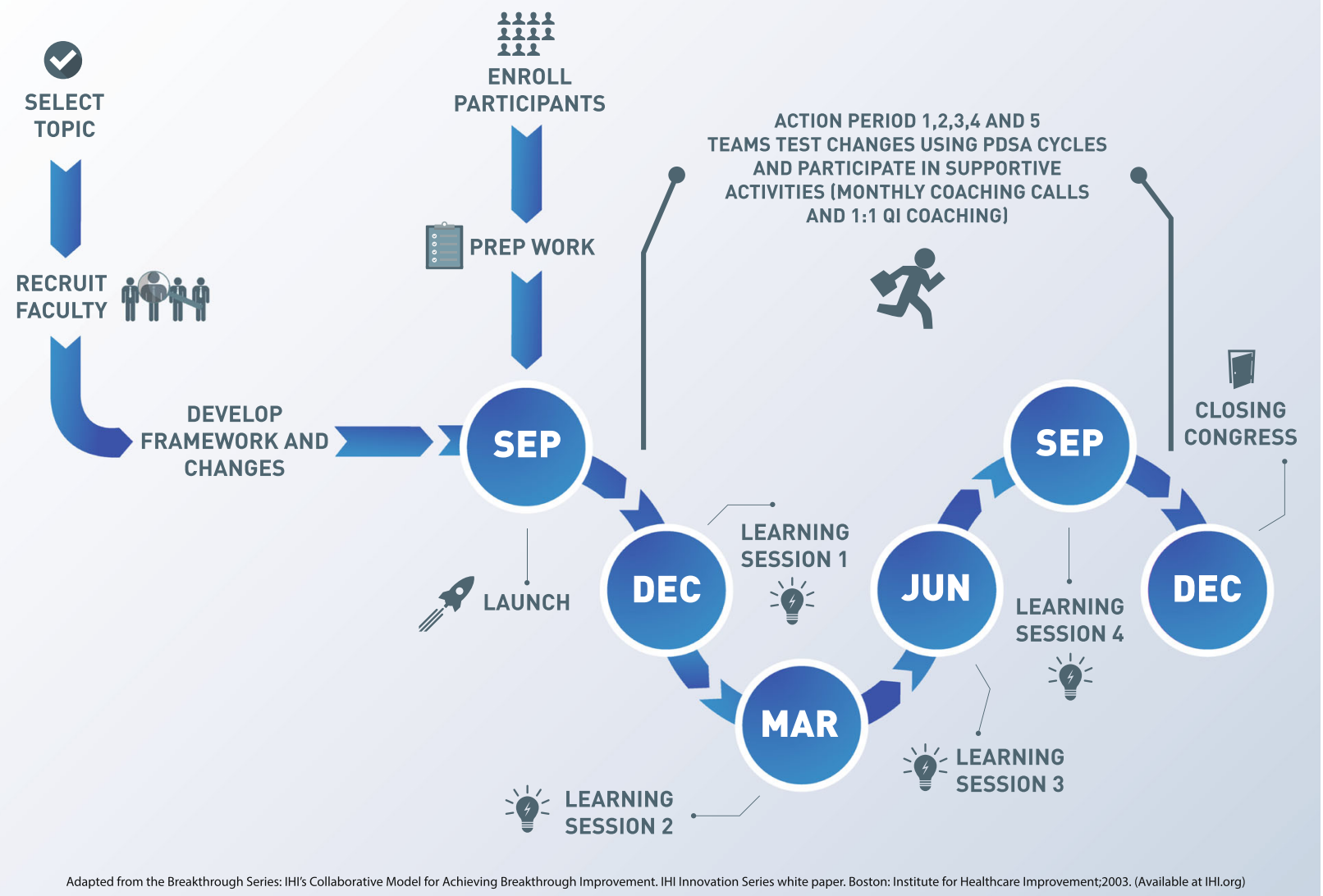

Fig. 1 Oral Opioid Agonist Therapy Collaborative Methodology 
BOOST Collaborative. The working group had representation from across programs and health disciplines in Vancouver Coastal Health along with representation from the community and people with lived experience. Directors from primary care, mental health and substance use programs in Vancouver Coastal Health were asked to identify programs that could participate in the Collaborative process, contribute to the shared aims, collect indicators and implement changes.

\section{Launch and preparation}

In August 2017, programs that were identified by leadership to participate, were invited to attend a 60-min informational webinar that aimed to introduce the purpose, aims and measurement strategy of the BOOST Collaborative. Program representatives attended the Collaborative Launch in September 2017 to orient them to the shared aims, technical content and expectations for participation. Participating programs consented by signing a letter of understanding before joining the Collaborative. Once program representatives were familiar with the Collaborative content, they were asked to form improvement teams and draft team-specific aims. Teams began to collect quality indicators by creating patient registries and standardizing their clinical data entry. Teams were provided with a series of evidence-based change ideas [27] that were aligned with CCM concepts ${ }^{8}$ (available online here: http://stophivaids.ca/STOP/wpcontent/uploads/post/BOOST-Collaborative-ChangePackage.pdf). Teams selected change ideas based on their local context, expertise and patient population.

\section{Support activities}

In-person learning sessions were held every three to four months bringing together representatives from each team. Participants learned about quality improvement, best-practices in OUD care and the provincial response to the overdose crisis. Participants also shared their own progress and learnings and were given dedicated time to plan for future tests of change.

Learning sessions were followed by action periods where teams conducted rapid tests of change using Plan-Do-Study-Act (PDSA) cycles and completed monthly quality indicator reports along with narrative descriptions of changes. Teams were supported with monthly educational webinars, a Collaborative electronic mailing list (listerv), monthly in-practice coaching visits by the core team (medical lead, Collaborative lead and QI coach), a website of resources, and targeted monthly team feedback.

The BOOST Collaborative staff met with Vancouver Coastal Health leadership every quarter to provide progress updates. Meetings were focused on highlighting improvement processes, support needed for teams, and team reported barriers and enablers.

\section{Measurement and evaluation \\ Data standardization}

The majority of teams participating in the BOOST Collaborative used the same electronic medical record (EMR) for patient charting that was capable of running data queries to extract aggregate QI metrics. Prior to implementing changes, teams were supported to standardize their clinical data entry through the use of an EMR form template. This form allowed the standardization of the following clinical information: International Classification of Diseases (ICD-9 version) diagnosis code, Most Responsible Provider, OAT type, daily dose, prescription start and end date, and treatment stage. The term treatment stage refers to the nature of the prescription for OAT for the client. Treatment stage can be characterized as a new start (first ever OAT prescription); re-start (OAT prescription after treatment interruption); dose increase; dose decrease; and dose unchanged.

\section{Quality indicators}

Teams reported monthly aggregated, non-nominal quality metrics and qualitative description of change from October 2017 to December 2018. Teams were expected to run queries in their EMR and extract the relevant quality indicators and transfer to a Microsoft Excel spreadsheet for reporting. Teams that did not use this EMR for patient charting were expected to manually extract relevant indicators and transfer to a Microsoft Excel spreadsheet for reporting. Qualitative descriptions of change were submitted on a Microsoft Word document. The rate of team participation in reporting was calculated by dividing the total number of times a team reported by 13 , the total number of reporting periods during the Collaborative.

Quality indicators were based on the cascade of OUD care in combination with current literature and input from the multi-stakeholder expert group. Indicators included: 1) engaged in care (a documented encounter with a primary care provider using the OUD form within the last 18 months); 2) OAT access (a documented prescription for OAT); 3) active OAT (have an active, nonexpired prescription for OAT); 4) retention in care $>3$ months; and 5) quality of life score (PROMIS Global 10) [28]. The impact of the changes was determined by averaging monthly quality indicators and plotting arithmetic means on run charts, a common tool used for visualizing improvement. A detailed description of the analysis is forthcoming. 


\section{Implementation scores}

From October 2017 to November 2018, teams were assigned an implementation score as a measure of progress in meeting their Collaborative aims and implementing changes. Scores ranged from 0.5 (no activity) to 5.0 (outstanding sustainable results) based on adapted Collaborative Assessment Scale criteria developed by the Institute for Health Care Improvement [29].

Implementation scores were determined by two reviewers. Monthly qualitative and quantitative reports were divided between the two reviewers and a score was applied. To ensure consistency between reviewers, the pair met monthly to review and discuss the assigned score. Final scores were applied after consensus between the two reviewers.

\section{Results}

\section{Participating teams}

Seventeen diverse health care teams participated in the BOOST Collaborative. Participating teams included 12 health authority owned and operated community health centres; two contracted primary health care agencies; one outreach focused team; and one rapid access SUD service and one HIV speciality clinic both located in a hospital setting. All teams were located in the Vancouver Community region (Downtown Vancouver, East Vancouver and South Vancouver). All teams were interdisciplinary with approximately five members per team and representation from key disciplines (e.g. nursing, medicine, social work and management).

Each team developed their own site-specific aim aligned with the overall Collaborative aims based on their population of focus and program mandate. Each team was encouraged to select from a series of evidencebased change ideas [27] (available online here: http://stophivaids.ca/STOP/wp-content/uploads/post/BOOST-

Collaborative-Change-Package.pdf) and were supported to run rapid tests of change (using PDSA cycles) and implement the changes if they were found to be successful. Evidence-based changes to improve access to care included identifying clients lost to care (no encounter for $>6$ months), improving intake forms (standardizing data entry), proactive monitoring and follow-up after missed doses of OAT, and adding reminder or follow-up calls for appointments.

\section{Client population}

A total of 4301 patients with OUD were identified as the population of focus within the 17 participating teams. The mean age of patients was $43(\mathrm{SD}=11)$ with $64 \%$ identifying as male, $35 \%$ female and $1 \%$ trans or nonbinary. The majority of patients lived in the Vancouver region (76\%) and approximately half of participants had a MRP assigned to them.

\section{Fidelity to BTS components}

The BOOST Collaborative closely followed the IHI's BTS Methodology, however small adaptations were accepted to support interdisciplinary participation and allow for site-specific customization. In some cases, team selection criteria were waived if a team could align itself with the overall Collaborative aims and demonstrate an ability to participate fully in the Collaborative process. For example, team selection criteria required the participation of an OAT prescriber (physician or nurse practitioner); however, this criterion was waived for an outreach-focused team made up of nurses and social workers, whose main goal was to provide outreach support following an overdose and connect those participants to care. Decisions regarding selection criteria were made by the medical lead and $\mathrm{VCH}$ project sponsors. In addition, all teams were supported with in-practice QI coaching, but the type and intensity of this coaching varied between teams. The adherence to the PDSA approach to testing changes also varied between teams. Finally, the 18-month timeline of the BOOST Collaborative is a modification to the traditional short-term BTS Collaborative methodology.

\section{Quality Indicator reporting}

Teams submitted qualitative and quantitative reports electronically each month to the Collaborative staff. Median qualitative reporting rate was $42 \%$ and ranged from 15 to $85 \%$ and the median quantitative reporting rate was $35 \%$ and ranged from 0 to $77 \%$.

\section{Implementation scores}

Fourteen of the 17 participating teams were given a monthly implementation score. Two of the 17 teams started receiving monthly implementation score on the third month of reporting and one of the teams started receiving implementation scores on the seventh month of reporting. This delay was due to challenges and limitations in data collection and capacity.

Median implementation scores rose from 2.0 in October 2017 to 3.0 in November 2018 (Fig. 2). Data showed that $41 \%$ of teams achieved a 2.5 (changes tested, but no improvement); 35\% achieved a 3.0 (modest improvement); and $24 \%$ achieved a 3.5 (improvement).

\section{Discussion}

From July 2017 to December 2018, 17 interprofessional healthcare teams were enrolled to participate in an 18month QI Collaborative. Participating teams made significant progress implementing evidence-based changes to clinic workflow and care processes with a focus on initiation and retention of OAT to improve outcomes for their patients with OUD. Changes to improve access to care included identifying clients lost to care, 
Collaborative Implementation Score

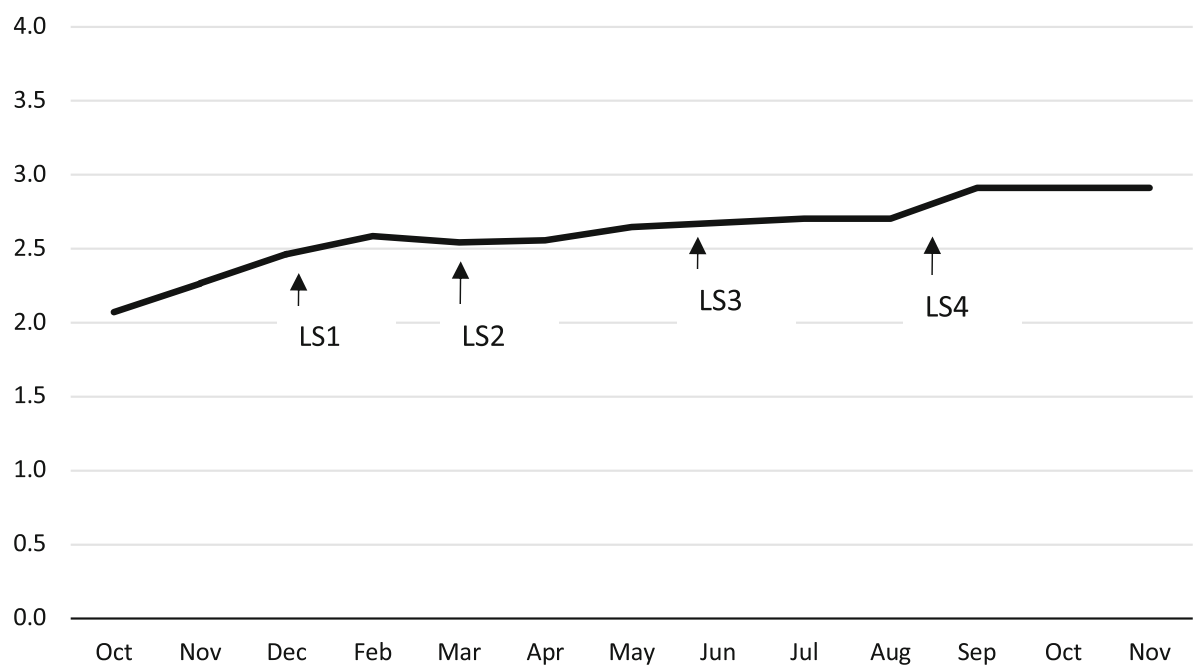

Fig. 2 Collaborative Implementation Scores

improving intake forms and adding reminder or followup calls for appointments. As a result, there was a significant increase in the number of individuals retained in OAT at the three-month mark from three out of ten to seven out of ten. Results from this study provide evidence to support the implementation QI and process improvement to improve retention on OAT for people living with OUD.

Improvements in care were captured in monthly reports containing process and outcome indicators and qualitative descriptions of change. Reporting rates were variable among teams with a median reporting rate of 42 and $35 \%$ for qualitative and quantitative reports, respectively. By reporting monthly on their progress, teams were able to determine if the changes they were implementing in practice, were indeed resulting in an improvement. Although reporting rates were variable among teams, this is consistent with other similar initiatives [12], and may reflect the reality that not all teams are able to establish a system of measurement to inform their quality improvement initiatives.

As part of the preparation phase for the BOOST Collaborative, the core team sought to address known issues with data quality in participating teams' EMRs. It has been shown that data quality can significantly influence the accuracy of quality indicators [30]; therefore, we aimed to improve the fidelity of clinical data as it was entered into the EMR. EMR forms have been found to improve the likelihood of patients receiving evidencebased care and the accuracy of both clinical and administrative data [31]. This is also in line with the CCM, which highlights the importance of decision support and information systems in the proactive care of patients with chronic conditions [8].
Monthly reports were used by the core team to assign a monthly Collaborative Assessment Score [29], a characterization of team progress throughout the Collaborative process. Overall, teams showed improved progress throughout the Collaborative, with the majority of teams (59\%) showing modest or some improvement. The relatively modest rates of improvement between teams may be explained by the challenges outlined above regarding data extraction and indicator reporting. Without demonstration of measurable improvement, teams cannot progress to a score greater than 2.5 (changes tested), limiting their ability to demonstrate improvements. Assessment scores do not fully capture the complexity of changes made and, when combined with quality indicator outcomes, can provide further information about team success and what may predict success for a participating team.

The BOOST Collaborative designed a QI initiative including a suite of OUD intervention strategies based on the HIV cascade of care [32]. This process requires taking a system-level view and systematically identifying gaps in care for people living with OUD from screening to diagnosis to follow-up. Further, it involves meaningfully including the client and family voice to characterize gaps in care and address this complex issue. The evidence clearly shows that people living with OUD have better health outcomes when engaged in care and retained in treatment, such as OAT [3, 4]. Retention in OAT is often suboptimal for a number of different reasons, including barriers to induction and titration, limited drop-in or clinic hours, and reactive patient recall systems. This initiative supported teams to more accurately identify and characterize patients with OUD (i.e., age, treatment stage, engagement in care and retention), 
and target interventions and changes based on that information to improve care and outcomes.

\section{Limitations and implications}

Unlike other similar QI initiatives, where healthcare teams go through an application process and pay to participate [12-14], Vancouver BOOST participants were selected by health authority leadership. This may have influenced team's receptiveness and readiness to participate in this initiative. Without an application process, there was a high variability of leadership support and commitment to quality improvement processes among teams. This may have impacted the ability of teams to engage fully in this work, particularly if time and resources were not consistently available to participants. Strong leadership commitment and financially accountable teams may have improved participants engagement in this initiative.

Although teams were provided with access to in-practice coaching and support to extract data from their EMRs, some teams had ongoing challenges with measurement and reporting and the workload it placed on staff. Qualitative data reporting rates ranged from 15 to $85 \%$ and quantitative data reporting rates ranged from 0 to $77 \%$. The differences in reporting rates may be partially explained by variable technical skills and comfort with EMR data and functionalities. Further to this, there were ongoing and, in some cases, significant challenges with EMR data quality, which created barriers to extracting useful quality indicator data, resulting in low reporting rates for some teams. A parallel commitment from service providers, vendors or internal information technology teams to support data fidelity may have improved this element of the Collaborative.

Baseline QI knowledge and uptake of QI resources was also highly variable among teams. Some teams had participated in previous QI Collaboratives and were very familiar with QI methodology and resources, whereas others had no knowledge at all. A considerable amount of time was dedicated up front to establishing a common understanding of QI among teams. Ongoing and ad-hoc training for new staff may have improved engagement from participating teams.

Other factors that may have influenced the success of implementation and team participation included a provincial system-wide primary care redesign initiative occurring in parallel with the BOOST Collaborative. This resulted in program reorganizations and restructuring, in addition to clinic moves. This was disrupting for many of the teams as staff and caseloads were redistributed.

Finally, implementing a QI Collaborative was very challenging in the midst of a public health emergency and substantial patient mortality. For participating teams from low-barrier clinics with no set appointment times, there were challenges related to protecting time for meetings and sourcing backfill staff to attend support activities given unpredictable and fluctuating demands. This initiative offers some encouraging results for improving care for people living with OUD; however, additional research is needed to determine the sustainability of these improvements beyond the end of the Collaborative.

\section{Conclusions}

The BOOST Collaborative developed a training program based on in-person and online learning sessions to translate evidence-informed best practices for treatment using OAT, introduce PDSA cycles for testing small changes in the clinical setting and create opportunities to share the successes and challenges of implementing these changes while striving for $95 \%$ retention on OAT. This initiative has successfully supported clinics to incorporate changes, such as adjusting clinic hours, increasing outreach capacity and structure, creating comfortable waiting rooms, engaging with pharmacies, incorporating the client/family voice as well as peers into the delivery model. Monthly qualitative and quantitative reports indicated that the majority of teams demonstrated some improvement using this intervention strategy.

Vancouver BOOST teams made significant progress in identifying areas of improvement and implementing changes with a focus on initiation of and retention on OAT. Changes to improve access to care included identifying clients lost to care, improving intake forms and adding reminder or follow-up calls for appointments. As a result, there was a significant increase in the number of individuals retained in OAT at the three-month mark. Results from this study support the feasibility of implementing an 18-month BTS quality improvement Collaborative in a publicly-funded health care system in Vancouver, Canada to improve access to care and retention on OAT.

\section{Abbreviations \\ BC: British Columbia; BC-CfE: B.C. Centre for Excellence in HIV/AIDS; BOOST: Best-practice in Oral Opioid agoniSt Therapy; BTS: Breakthrough series; CCM: Chronic care model; EMR: Electronic medical record; ICD- 9: International Classification of Diseases; $\mid H I$ : Institute for Healthcare Improvement; MRP: Most responsible provider; OAT: Opioid agonist therapy; OUD: Opioid use disorder; PDSA: Plan-Do-Study-Act; QI: Quality improvement; SD: Standard deviation; SUD: Substance Use Disorder; VCH: Vancouver Coastal Health}

\section{Acknowledgements}

We would like to thank Angie Semple for her administrative support throughout the collaborative and the Practice Support Program for their coaching support. Thank-you to all the managers and directors at Vancouver Coastal Health and the BC-CfE who supported the work of the collaborative. Finally, thank-you to all the physicians, nurses, social workers, counsellors and staff who participated in the Collaborative and made this work possible.

\section{Authors' contributions}

$\mathrm{LB}$ analyzed and interpreted the data regarding the implementation and wrote a first draft of the manuscript. ZS, CS, KE, RG, DC, RB and JK were 
involved in the enactment of the initiative and its evaluation, and were major contributors in revising the manuscript. All authors read and approved the final manuscript.

\section{Funding}

The BOOST Collaborative was funded by the BC-CfE and VCH. Dr. Rolando Barrios, Senior Medical Director at VCH and Assistant Director at the BC-CfE represented the funding bodies and provided guidance throughout the design and development of the manuscript and input and feedback on the manuscript drafts. Dr. Klimas' work on this paper was supported, in part, by a grant from European Commission (701698).

\section{Availability of data and materials}

The data that support the findings of this study are available from the corresponding author, RB, upon reasonable request. However, note that participating teams only reported aggregated, non-nominal data.

\section{Ethics approval and consent to participate}

The study met the Providence Healthcare Research Ethics Board criteria for quality improvement initiatives and, therefore, has been exempted from review. Under Article 2.5 of the Tri Council Policy Statement of the Canadian Institutes of Health Research Natural Sciences, Engineering Research Council of Canada Social Sciences and Humanities Research Council of Canada, Quality Improvement activities are not subject to institutional review. Collaborative teams consented by signing a letter of understanding before joining the Collaborative.

\section{Consent for publication}

Not applicable.

\section{Competing interests}

None reported.

\section{Author details}

${ }^{1}$ British Columbia Centre for Excellence in HIV/AIDS (BC-CfE), St. Paul's Hospital, 608-1081 Burrard Street, Vancouver, BC V6Z1Y6, Canada. Vancouver Coastal Health, 520 W 6th Ave, Vancouver, BC V5Z4H5, Canada. 'St. Paul's Hospital, Providence Health Care, 608-1081 Burrard Street, Vancouver, BC V6Z1Y6, Canada. "British Columbia Centre on Substance Use, 400-1045 Howe St, Vancouver, BC V6Z2A9, Canada. ${ }^{5}$ Faculty of Medicine, University of British Columbia, 2206 East Mall, Vancouver, BC V6T1Z3, Canada. ${ }^{6}$ Department of Medicine, University of British Columbia, St. Paul's Hospital, 608-1081 Burrard Street, Vancouver, BC V6Z1Y6, Canada. ${ }^{7}$ School of Medicine, Health Sciences Centre, University College Dublin, Belfield, Dublin 4, Ireland.

Received: 30 April 2019 Accepted: 27 August 2019

Published online: 14 September 2019

\section{References}

1. Government of $B C$ coroners service. Illicit drug overdose deaths in $B C$ January 1, 2009 - January 31, 2019 Government of British Columbia 2019.

2. Bruneau J, Goyer M, Ahamad K, Poulin G, Selby P, Fischer B, et al. Management of opioid use disorders: a national clinical practice guideline. Can Med Assoc J. 2018;190(9):E24-E257.

3. Sordo L, Barrio G, Bravo MJ, Indave BI, Degenhardt L, Wiessing L, et al. Mortality risk during and after opioid substitution treatment: systematic review and meta-analysis of cohort studies. BMJ. 2017;26:357-1550.

4. Mattick RP, Breen C, Kimber J, Davoli M. Buprenorphine maintenance versus placebo or methadone maintenance for opioid dependence. The Cochrane database of systematic reviews. 2014;6(2):CD002207.

5. Reist D. Methadone maintenance treatment in British Columbia, 1996-2008: analysis and recommendations; 2010.

6. Alford DP, LaBelle CT, Kretsch N, Bergeron A, Winter M, Botticelli M, et al. Collaborative Care of Opioid-Addicted Patients in primary care using buprenorphine: five-year experience. Arch Intern Med. 2011;171(5):425-31.

7. Canadian Medical Protective Agency. Who is the most responsible physician? Check your knowledge. 2016; Available at: https://www.cmpaacpm.ca/en/advice-publications/cmpa-perspective/2016/who-is-the-mostresponsible-physician-check-your-knowledge. Accessed June 18, 2019.

8. Chronic Disease Prevention. A framework for a provincial chronic disease prevention initiative. Ministry of Health 2003 October.
9. Batalden PB, Davidoff F. What is "quality improvement" and how can it transform healthcare? Quality and Safety in Health Care. 2007 Feb;16(1):2-3.

10. The Institute for Healthcare Improvement. The breakthrough series: IHI's collaborative model for achieving breakthrough improvement: Institute for Healthcare Improvement; 2003.

11. Schouten LMT, Hulscher MEJL, van Everdingen JJE, Huijsman R, Grol RPTM. Evidence for the impact of quality improvement collaboratives: systematic review. BMJ. 2008;336(7659):1491-4.

12. Hoffman KA, Ford JH, Choi D, Gustafson DH, McCarty D. Replication and sustainability of improved access and retention within the network for the improvement of addiction treatment. Drug Alcohol Depend. 2008;98(1):63-9.

13. McCarty D, Gustafson D, Capoccia V, Cotter F. Improving Care for the Treatment of alcohol and drug disorders. Journal of Behavioural Health Services Research. 2009;36(1):52-60.

14. Gustafson DH, Quanbeck AR, Robinson JM, Ford JH, Pulvermacher A, French MT, et al. Which elements of improvement collaboratives are most effective? A cluster-randomized trial. Addiction. 2013;108(6):1145-57.

15. The Ministry of Health. Preventing and Managing Diabetes in British Columbia. Government of British Columbia 2007.

16. Healthy Heart Society of British Columbia. Province-wide CHF Collaborative. 2004; Available at: http://www.ihi.org/resources/Pages/ImprovementStories/ ProvincewideCHFCollaborative.aspx. Accessed June 28, 2019.

17. Clarke CM, Cheng T, Reims KG, Steinbock CM, Thumath M, Milligan RS, et al. Implementation of HIV treatment as prevention strategy in 17 Canadian sites: immediate and sustained outcomes from a 35-month quality improvement collaborative. BMJ Quality and Safety. 2016;25(5):345-54.

18. Government of BC. Census Population of BC and Canada 2011; Available at: http://www.bcstats.gov.bc.ca/StatisticsBySubject/Census/2011Census.aspx.

19. Government of BC. Provincial health officer declares public health emergency. 2016; Available at: https://news.gov.bc.ca/releases/2016 HLTH0026-000568. Accessed March 26, 2019.

20. Office of the Provincial Health Officer. BC Opioid Substitution Treatment System Performance Measures 2015/2015 - 2015-2016. Government of British Columbia 2017 March

21. Government of British Columbia. Responding to B.C.'s Illegal Drug Overdose Epidemic Progress Update; Government of British Columbia 2018 April.

22. Egilson M. BC coroners service death review panel. British Columbia Coroners Service; 2018.

23. Bellringer C. Health funding explained, vol. 2: Office of the Auditor General of British Columbia; 2017.

24. Government of BC. Psychiatric Medications Plan (Plan G). 2016; Available at: https:// wuw2.gov.bc.ca/gov/content/health/practitioner-professional-resources/pharmacare/ prescribers/psychiatric-medications-plan-plan-g. Accessed March 25, 2019.

25. Vancouver Coastal Health. Sessional Rates. 2019; Available at: http://medicalstaff.vch. ca/working-at-vch/compensation/sessional-rates/. Accessed march 26, 2019.

26. Providence Health Care. John Ruedy Clinic JRD. http://jrc. providencehealthcare.org/. Accessed April 19, 2019.

27. BC Centre for Excellence in HIV/AIDS. BOOST Collaborative. http:// stophivaids.ca/oud-collaborative/. Updated 2017. Accessed April 29, 2019.

28. US Department of Human and Health Services. PROMIS health measures. http://www.healthmeasures.net/explore-measurement-systems/promis. Updated 2019. Accessed June 18, 2019.

29. Institute for Healthcare Improvement. Assessment Scale for Collaboratives. http://www.ihi.org/resources/Pages/Tools/AssessmentScaleforCollaboratives aspx. Updated 2004. Accessed August 2, 2019

30. Dentler K, Cornet R, ten Teije A, et al. Influence of data quality on computed Dutch hospital quality indicators: a case study in colorectal cancer surgery. BMC Medical Informatics and Decision Making. 2014;14(1):32.

31. Herrin J, da Graca B, Aponte P, et al. Impact of an EHR-based diabetes management form on quality and outcomes of diabetes care in primary care practices. Am J Med Qual. 2015;30(1):14-22.

32. Nosyk B, Montaner JSG, Colley G, Lima V, Chan K, Heath K, Yip B, Samji H, Gilbert M, Barrios R, Gustafson R, Hogg RS. The cascade of HIV care in British Columbia, Canada, 1996-2011: a population-based retrospective cohort study. Lancet Infect Dis. 2014;14(1):40-9.

\section{Publisher's Note}

Springer Nature remains neutral with regard to jurisdictional claims in published maps and institutional affiliations. 